Conclusion Lipid profile changes in HGSC may have considerable prognostic value for the disease after treatment. The signatures defined by our work may provide a basis for the development of prognostic tools and may predict the clinical course of HGSC patients.

This work was supported by RSF grant $\square$ 20-65-46014. Disclosures Nothing to discloser.

\section{CAN D-DIMERS AND FIBRINOGEN AID IN THE DIAGNOSIS OF OVARIAN CANCER?}

${ }^{1}$ Kate Mckendry, ${ }^{2}$ Sharon O'toole. 'St James's Hospital; ${ }^{2}$ Department of Obstetrics and Gynaecology; Trinity College Dublin

\subsection{6/ijgc-2020-ESGO.103}

Introduction/Background Ovarian cancer (OC) mortality rates remain high due to a lack of early predictive biomarkers. Elevated levels of fibrinogen and its end product D-dimers are found in OC compared to benign controls and can predict poor prognosis independent of venous thromboembolism. Limited studies examining fibrinogen and D-dimers separately alongside CA125 suggest they may be of use in differentiating benign from malignant disease however no clear conclusions have been drawn due to a lack of prospective trials. The aim of this study was to evaluate the performance of D-dimers and fibrinogen alongside established predictors of OC, either alone or in combination, compared to CA125 alone.

Methodology Pre-operative serum samples were collected from 296 patients undergoing primary debulking surgery for pelvic masses. Levels of CA125, D-dimers, fibrinogen, Human Epididymis Protein 4 (HE4), the Risk of Ovarian Malignancy Algorithm, and the Risk of Malignancy Index I and II were assessed and sensitivity and specificity calculated. Logistic regression models were fitted for each individual biomarker and for various biomarker combinations and an ROC comparison was performed.

Results The study consisted of 96 pre- and 200 post-menopausal women with 154 benign, 43 borderline, and 99 malignant cases. CA125, D-dimers and fibrinogen had sensitivities of $73 \%, 75 \%$ and $65.2 \%$ and specificities of $69.5 \%, 55.9 \%$ and $57.6 \%$, respectively. The combination of HE4 and fibrinogen had the highest ROC-AUC in the premenopausal group at $75.8 \%$ but no biomarker(s) reached statistical significance compared to CA125. In the postmenopausal group, two panels $(\mathrm{CA} 125+\mathrm{HE} 4+\mathrm{D}$-dimer + fibrinogen, and HE4 $+\mathrm{d}$ dimer + fibrinogen) were significantly different to CA125 on ROC analysis (both $\mathrm{p}=0.029$ ).

Conclusion As individual biomarkers, D-dimers and fibrinogen are both limited by poor specificity using general population cut-offs. Where these biomarkers may play a role in OC is as part of biomarker panels. We developed a highly accurate multivariable model including HE4, d-dimers and fibrinogen which improved the diagnostic accuracy of CA125 alone in postmenopausal women. In pre-menopausal women, fibrinogen may play a role alongside HE4 as a second-step test, when imaging is inconclusive and CA125 is positive, to increase specificity.

Disclosures None.

\section{RISK FACTORS FOR PREOPERATIVE COMPLICATIONS AND MANAGEMENT WITH ENHANCED RECOVERY AFTER PRIMARY SURGERY FOR WOMEN WITH EPITHELIAL OVARIAN CANCER IN A SINGLE CENTER OF CHINA}

${ }^{1}$ Ying Zhou, ${ }^{2}$ Chenchen Zhu, ${ }^{3}$ Zhen Shen, ${ }^{3}$ Yanhu Xie, ${ }^{4}$ Wei Zhang, ${ }^{3}$ Tianjiao Zhang, ${ }^{3} \mathrm{LI}$ Min, ${ }^{3}$ Weidong Zhao, ${ }^{3}$ Dadao Wu, ${ }^{5}$ Björn Nashan. ${ }^{1}$ Department of Obstetrics and Gynecology, The First Affiliated Hospital of Ustc, Division of Life Sciences and Medicine, University of Science and Technology of China; ${ }^{2}$ Department of Obstetrics and Gynecology, Anhui Provincial Hospital, Anhui Medical University; ${ }^{3}$ The First Affiliated Hospital of Ustc, Division of Life Sciences and Medicine, University of Science and Technology of China; Department of Obstetrics and Gynaecology; ${ }^{4}$ The First Affiliated Hospital of Ustc, Division of Life Sciences and Medicine, University of Science and Technology of China; Department of Anaesthesiology; ${ }^{5}$ The First Affiliated Hospital of Ustc, Division of Life Sciences and Medicine, University of Science and Technology of China; Organ Transplantation Center

10.1136/ijgc-2020-ESG0.104

Introduction/Background This study aimed to evaluate the postoperative complications and their impacts on patients who have undergone primary surgery (including extensive upper abdominal surgery) of ovarian epithelial cancer with the enhanced recovery programme.

Methodology We identified all patients with stage I $\square$ IV ovarian carcinoma who underwent primary surgery in our centre. Postoperative complications were evaluated and graded according to the Clavien-Dindo Classification.

Results Among 161 patients, 46 cases (28.57\%) were performed with surgical staging, $27(16.77 \%)$ with standard cytoreduction, $12(7.45 \%)$ with en-bloc debulking, and 76(47.20\%) with extraradical debulking; 157 patients (97.52\%) achieved optimal tumour reduction $(<1 \mathrm{~cm})$. The mean postoperative hospitalisation time was $17.33 \pm 11.29$ days after completion of initial postoperative chemotherapy (IPC), and the interval of IPC was $16.22 \pm 10.09$ days. Thirteen patients $(8.07 \%)$ had grade 3 complications (9 wound dehiscence, 3 digestive tract leakage, and 1 bladder fistula). Two patients (1.24\%) had grade 4-5 complications (1 severe pneumonia infection and back to intensive care unit [ICU] for tracheotomy and respiration rehabilitation; 1 died of septicaemia on day 19). As for preoperative factors analysis, multivariate analysis revealed that HE4 $\geq 717 \mathrm{pM}(\mathrm{P}=0.015)$ and Federation International of Gynecology and Obstetrics (FIGO) IV stage ( $\mathrm{P}=0.004$, compared with IIIC stage) were associated with grade $3 \square 5$ complications. Bootstrap analysis found CA125 $\geq 1012 \mathrm{U} / \mathrm{mL}(\mathrm{P}=$ $0.034)$, HE4 $\geq 717 \mathrm{pM}(\mathrm{P}=0.007)$, and FIGO IV stage ( $\mathrm{P}$ $=0.002$, compared with IIIC stage) had statistical significance. As for postoperative factors analysis, multivariate analysis did not reveal the risk factors associated with grade $3 \square 5$ complications; bootstrap analysis only found that transfer to ICU after surgery $(\mathrm{P}=0.026)$ had statistical significance.

Conclusion Application of enhanced recovery after surgery protocols in epithelial ovarian carcinoma are useful and support the early initiation of chemotherapy and a short hospitalisation time, and it is safe for primary extensive radical cytoreduction with low mortality $1 / 76$ (1.31\%).

Disclosures This study was funded by the National Natural Science Foundation of China (81872110, 81902632), National Key R\&D Program of China (2018YFC1003900), the Anhui Provincial Innovative Program for Organ Transplantation (S20183400001), and Anhui Provincial Key Research and Development Program (1704a0802151). The funders had no 
role in the study design, data collection and analysis, decision to publish, or preparation of the manuscript. The authors declare that they have no competing interests.

\section{PRELIMINARY RESULTS OF ANLOTINIB AND NIRAPARIB DUAL THERAPY EVALUATION IN PLATINUM-RESISTANT RECURRENT OVARIAN CANCER (ANNIE): A MULTICENTER, SINGLE-ARM, PHASE 2 TRIAL}

${ }^{1}$ Guochen Liu, ${ }^{1}$ Jihong Liu, ${ }^{2}$ Bingna Xian, ${ }^{3}$ Yanling Feng, ${ }^{3}$ Qidan Huang. ${ }^{1}$ Sun Yat-Sen University Cancer Center; Department of Gynecologic Oncology; ${ }^{2}$ Sun Yat-Sen University Cancer Center; ${ }^{3}$ Department of Gynecologic Oncology

\subsection{6/ijgc-2020-ESG0.105}

Introduction/Background Patients with platinum-resistant ovarian cancer have a poor prognosis. Effective treatment options for these patients are limited. In this study (ANNIE), we evaluate the activity of niraparib combined with anlotinib in patients with platinum resistant recurrent ovarian carcinoma.

Methodology The ANNIE trial was a multicentre, single-arm, phase 2 study that evaluated the safety and activity of niraparib combined with anlotinib in patients $(\geq 18 \& \leq 70$ years) with recurrent ovarian epithelial, fallopian tube, or primary peritoneal cancer cancer whose disease recurred in less than 6 months after the last administered platinum therapy. Patients received oral niraparib $300 \mathrm{mg} / 200 \mathrm{mg}$ once daily continuously and anlotinib 12 mg on day 1-14 of each 21-day cycle thereafter until disease progression or intolerable toxicity. The primary objective was to assess objective response rate (ORR; complete plus partial responses). 40 cases are planned to be enrolled.

Results Between May 22, 2020 and August 3, 2020, we enrolled 9 patients (median age, 54 years [range, 44-64 years]). Patients had received a median of five (range, 2-8) previous lines of therapy. All but one (voluntarily withdrew) of the patients were still on treatment, the longest has been taking medication for more than 4 cycles. Three patients underwent imaging evaluation, including 1 confirmed complete responses, 1 with confirmed partial responses, 1 with stable disease. No drug-related grade 3 or worse treatment-emergent adverse events were detected, the most common treatment emergent adverse events were hypertension (5 of 9 patients), hand-foot skin reaction (4 of 9 patients), hoarseness (4 of 9 patients). Enrollment was ongoing so far.

Conclusion It seems niraparib in combination with anlotinib is tolerable, with promising antitumor activity for patients with platinum resistant recurrent ovarian cancer. Besides, we observed unusual safety signals in the combination (more hoarseness and less haematological toxicities). The conclusion can be clarified after the research is completed.

Disclosures Trial registration ClinicalTrials. gov identifier: NCT04376073, Funding: Zai Lab, The authors declare no conflicts of interest.

\section{\begin{tabular}{l|l}
247 & OPTIMISING PREDICTION ACCURACY OF COMPLETE
\end{tabular} CYTOREDUCTION FOR HIGH GRADE SEROUS ADVANCED OVARIAN CANCER PATIENTS USING NEAREST-NEIGHBOR MODELS}

Alexandros Laios, Yong Tan, Richard Hutson, George Theophilou, Diederick Dejong. St James's University Hospital; Leeds Teaching Hospitals; Gynaecologic Oncology
Introduction/Background The foundation of modern ovarian cancer care is cytoreductive surgery to remove all macroscopic disease (R0). Identification of R0 resection patients may help individualise treatment. Machine learning and AI have been shown to be effective systems for classification and prediction. For a disease as heterogenous as ovarian cancer, they could potentially outperform conventional predictive algorithms for routine clinical use. We investigated the performance of an $\mathrm{AI}$ system, the k-nearest neighbor (k-NN) classifier to predict R0, comparing it with logistic regression.

Methodolog A cohort of patients diagnosed with high grade serous advanced ovarian, tubal and primary peritoneal cancer (HGSOC), undergoing surgical cytoreduction from 20152019, was selected from the ovarian database. Performance variables included age, BMI, Charlson Comorbidity Index (CCI), timing of surgery, surgical complexity and disease scores. The k-NN algorithm classified R0 vs non-R0 patients using 3-20 nearest neighbors. Prediction accuracy was estimated as percentage of observations in the training set correctly classified. The relative importance of the selected variables was quantified by calculating the prediction accuracy/ error rate in relation to the number of predictors included in the models.

Results 154 patients were identified, with mean age of $64.4+$ 10.5 yrs, BMI of $27.2+5.8$ and mean SCS of $3+1$ (1-8) Complete and optimal cytoreduction was achieved in $62 \%$ and $88 \%$ patients. The mean predictive accuracy was $66 \%$ compared to $63.4 \%$ by logistic regression. R0 resection prediction of true negatives was as high as $90 \%$ using $\mathrm{k}=20$ neighbors. From the variables tested to contribute in R0 prediction, only disease score was statistically significant $(\mathrm{p}=0.0006)$. For a given neighborhood size $\mathrm{k}=15$, $\mathrm{R} 0$ resection was best

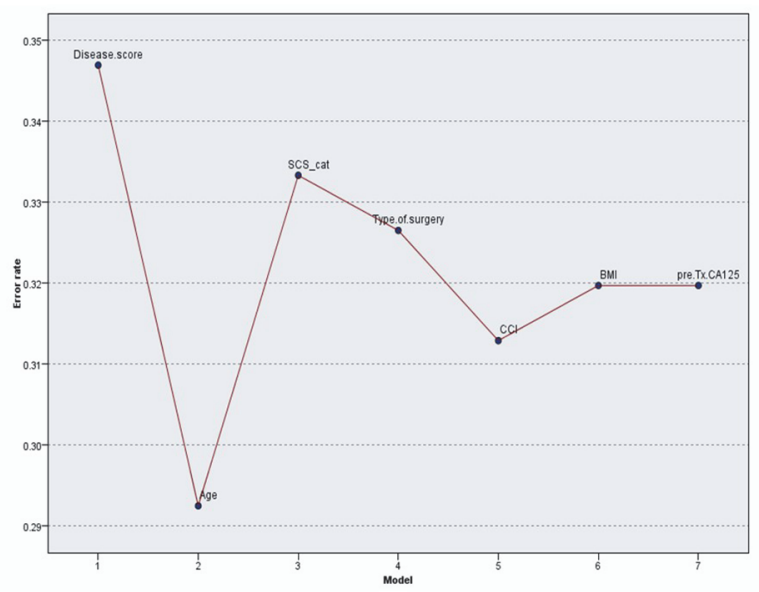

Abstract 247 Figure 1

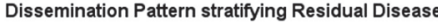

R1 R0

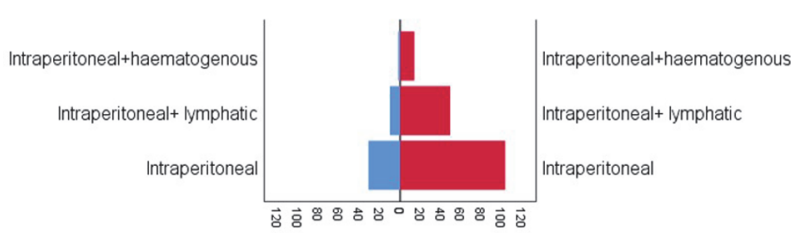

Abstract 250 Figure 1 